\title{
The PulseCath iVAC 2L left ventricular assist device: conversion to a percutaneous transfemoral approach
}

Nicolas M. Van Mieghem ${ }^{1 *}$, MD, PhD; Joost Daemen ${ }^{1}$, MD, PhD; Mattie J. Lenzen ${ }^{1}, \mathrm{PhD}$; Rob Zandstra ${ }^{2}$, MSc; Oren Malkin, MBA; Robert-Jan M. van Geuns ${ }^{1}, \mathrm{MD}, \mathrm{PhD}$

1. Department of Cardiology, Thoraxcenter, Erasmus Medical Center, Rotterdam, The Netherlands; 2. PulseCath BV, Arnhem, The Netherlands

This paper also includes supplementary data published online at: http://www.pcronline.com/eurointervention/90th_issue/168

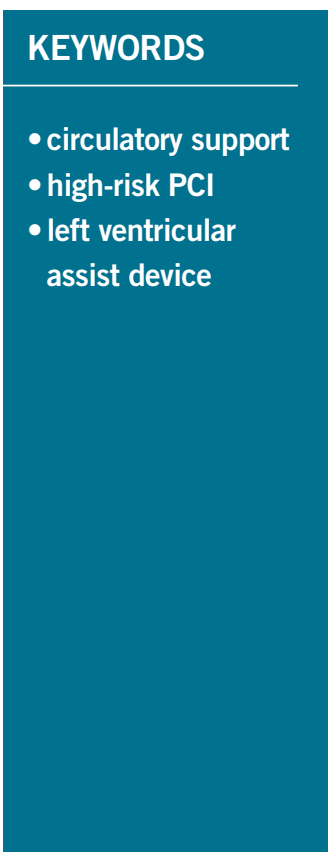

\section{Abstract}

Aims: This technical report describes the essentials and practical implantation technique of the completely percutaneous PulseCath iVAC 2L left ventricular assist device.

Methods and results: Percutaneously inserted mechanical left ventricular assist devices are used for circulatory support during cardiogenic shock or high-risk percutaneous coronary interventions. The PulseCath concept is a novel pulsatile design that consists of an extracorporeal membrane pump connected to a largebore catheter which is inserted across the aortic valve retrogradely into the left ventricle. A genuine intraaortic balloon pump (IABP) console drives the pulsatile pump.

Conclusions: The percutaneous PulseCath iVAC $2 \mathrm{~L}$ is driven by a genuine IABP console and provides more left ventricular support.

*Corresponding author: Department of Interventional Cardiology, Thoraxcenter, Erasmus MC, Room Bd 171, 's Gravendijkwal 230, 3015 CE Rotterdam, The Netherlands. E-mail: n.vanmieghem@erasmusmc.nl 


\section{Introduction}

Percutaneously inserted mechanical left ventricular assist devices (LVAD) are used for circulatory support during cardiogenic shock or high-risk percutaneous coronary interventions ${ }^{1-3}$. Cardiology and cardiac surgery societies on both sides of the Atlantic have issued joint guidelines on myocardial revascularisation and myocardial infarction, including prudent recommendations on the use of LVADs ${ }^{4,5}$. Data to support LVADs in patients with cardiogenic shock or undergoing high-risk PCI are scarce, yet these devices remain an essential part of the complete armamentarium of a tertiary care facility dealing with challenging patients who have complex coronary pathology. Compared with continuous flow systems, pulsatile LV support results in higher systemic perfusion pressure, theoretically augmented coronary perfusion and more unloading of the right heart and pulmonary circulatory system ${ }^{6}$.

The net haemodynamic benefits with the intra-aortic balloon pump (IABP) appear modest at best, with a cardiac output increase up to $0.5 \mathrm{~L} / \mathrm{min}^{7}$. The iVAC $3 \mathrm{~L}^{\mathrm{TM}}$ (PulseCath BV, Amsterdam, The Netherlands) is a membrane pump connected to a 21 Fr catheter that is inserted across the aortic valve retrogradely into the left ventricle: it is driven by a genuine IABP console and can generate a pulsatile blood flow up to $3 \mathrm{~L} / \mathrm{min}^{8}$. The PulseCath iVAC $3 \mathrm{~L}$ requires surgical access to the right subclavian artery. This technical report illustrates the newest-generation PulseCath iVAC $2 \mathrm{~L}$ and its conversion to a true percutaneous transfemoral LVAD.

\section{Technical specifications DESCRIPTION}

The PulseCath iVAC 2L has three essential components: 1) the membrane pump, 2) a bi-directional flow catheter, and 3) a patented rotating two-way valve (Figure 1, Figure 2). The extracorporeal membrane pump is transparent and contains a blood

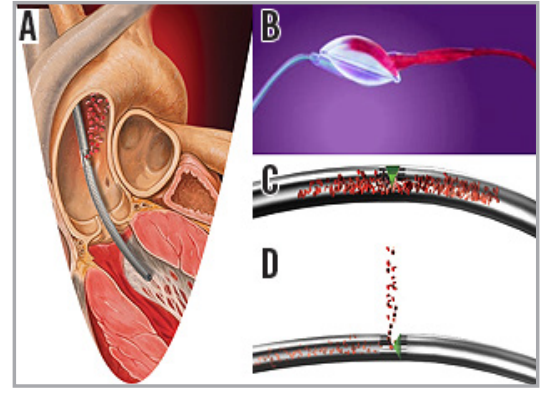

Figure 2. Cartoon illustration of the PulseCath iVAC 2L. A) Catheter across the aortic valve with its distal tip in the left ventricle and expulsion of blood above the aortic valve into the ascending aorta. B) Extracorporeal transparent membrane pump with blood entering the blood chamber. C) Bi-directional flow catheter with the valve (in green) closed towards the ascending aorta; blood will be directed from the left ventricle to the extracorporeal pump. D) Bi-directional flow catheter with the valve (in green) open to the ascending aorta; blood will be directed from the extracorporeal pump to the ascending aorta.

chamber and an air chamber divided by a thin flexible membrane. The blood chamber is connected to the bi-directional flow catheter and the air chamber to a mainstream IABP console, which acts as the pneumatic driver of the pump (Moving image 1). The total chamber volume is $40 \mathrm{~mL}$. The pump can expel a maximum volume of blood of $21 \mathrm{ml}$. The bi-directional flow catheter is composed of nitinol wire-reinforced polyurethane, measures $95 \mathrm{~cm}$ and has a $17 \mathrm{Fr}(5.9 \mathrm{~mm})$ outer diameter. The inlet tip is made of stainless steel. At $6 \mathrm{~cm}$ from the inlet tip, the catheter has an integrated two-way valve that pivots around two axes. A connector piece at the other end of the catheter connects to the membrane pump.

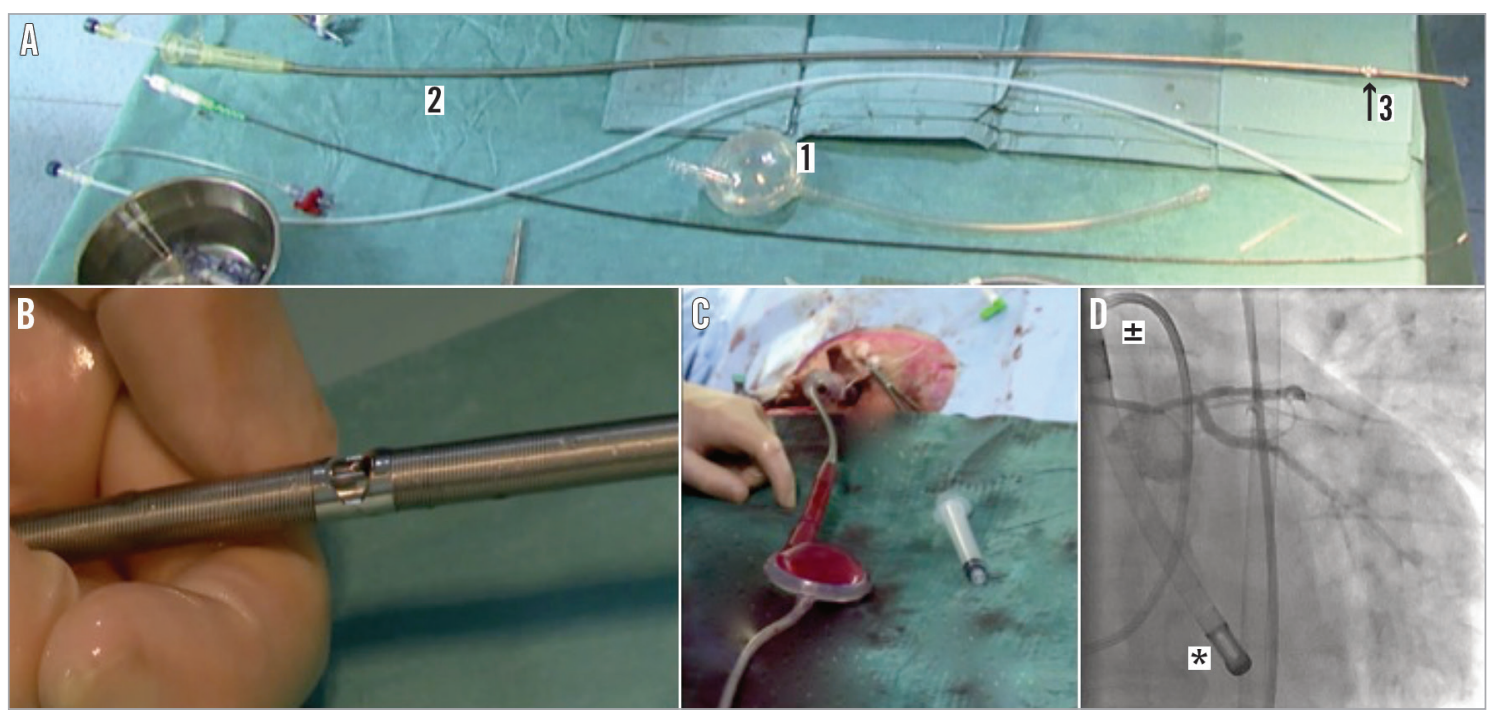

Figure 1. The PulseCath iVAC 2L. A) Extracorporeal transparent membrane pump with a blood chamber and an air chamber divided by a thin flexible membrane (1), bi-directional flow catheter (2), and rotating two-way valve (3). B) Close-up of the rotating two-way valve. C) Extracorporeal transparent membrane pump filled with blood. D) PulseCath iVAC $2 L$ in situ with the distal tip (*) in the left ventricle and the two-way valve above the aortic valve ( \pm ). 


\section{CONCEPT}

The catheter is inserted through the common femoral artery. The catheter is advanced retrogradely across the aortic valve into the left ventricle. The catheter is de-aired and connected to the extracorporeal membrane pump, which itself is connected to the IABP console. Using ECG triggering, blood is aspirated from the left ventricle through the inlet tip into the membrane pump during systole and ejected from the pump through the catheter valve into the ascending aorta during diastole. The pivotal valve function at the outflow port is synchronised with aortic valve closure and precludes interference with aortic valve functioning. The resulting pulsatile support generates additional output with (analogous to a typical IABP) systolic unloading of the left ventricle and diastolic counterpulsation (Figure 3 ).

\section{HAEMODYNAMIC EFFECTS}

The PulseCath iVAC 2L can expel up to $21 \mathrm{~mL}$ per beat. At higher heart rates $(>80 \mathrm{bpm})$ the net stroke volume will drop because the diastolic phase decreases and the pump no longer has sufficient time to eject its maximum volume. The iVAC $2 \mathrm{~L}$ can generate a maximum output of $2.2 \mathrm{~L} / \mathrm{min}$ in vitro. In vivo the pump generates an output of up to $1.5 \mathrm{~L} / \mathrm{min}$. Figure 4, Figure 5 and Figure 6 illustrate the typical haemodynamic changes during PulseCath iVAC 2L support. The mean systemic arterial pressure and cardiac output increase; mean pulmonary arterial pressure and mixed venous oxygen saturation remain stable. Of note, no significant haemolysis is perceived.

\section{Implantation step by step PREPARATION}

The PulseCath iVAC 2L requires an $18 \mathrm{Fr}$ femoral sheath. A thorough knowledge of the iliofemoral arterial tree is thus crucial to confirm eligibility and avoid access-site complications. Conventional contrast angiography or even a multi-slice computed tomography (MSCT) scan à blanc without contrast may prove to be a valuable screening tool. The minimum lumen arterial diameter should exceed $6 \mathrm{~mm}$ to accommodate the $18 \mathrm{Fr}$ sheath. Furthermore, excessive tortuosity and vessel wall calcification should be avoided. Multiple access techniques are possible

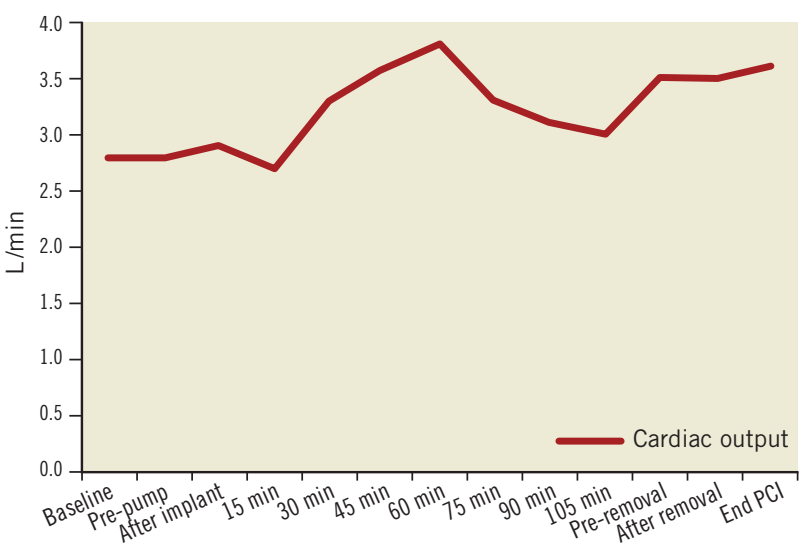

Figure 4. Change in cardiac output with PulseCath iVAC $2 L$ support.

including fluoroscopy-guided puncture and use of a landmark pigtail coming from the contralateral side. We prefer echo-guided access to guarantee access above the femoral bifurcation, avoid calcified regions and posterior wall puncture, and minimise radiation exposure to patient and operator. We first introduce a $6 \mathrm{Fr}$ arterial sheath and make a contrast angiogram to confirm appropriate access and vessel characteristics. We then use a pre-closure technique with either one $\operatorname{Prostar}^{\circledR}$ or two ProGlides ${ }^{\circledR}$ (Abbott

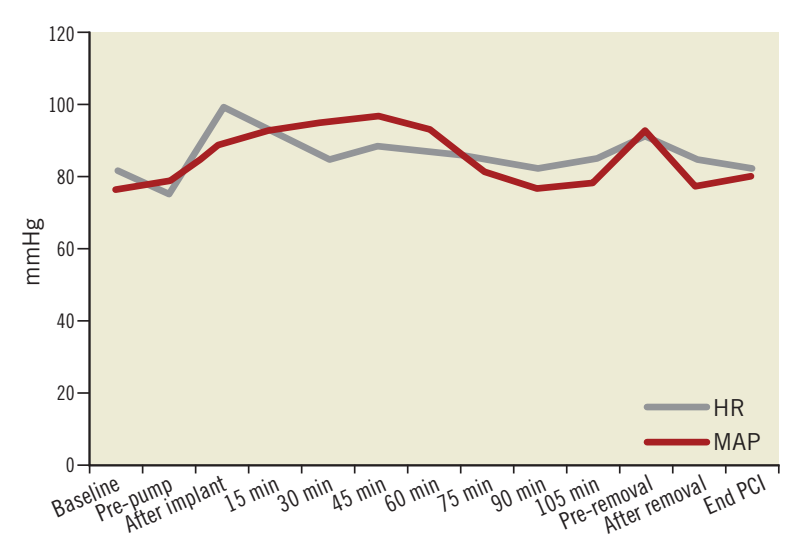

Figure 5. Change in heart rate (HR) and mean arterial pressure (MAP).

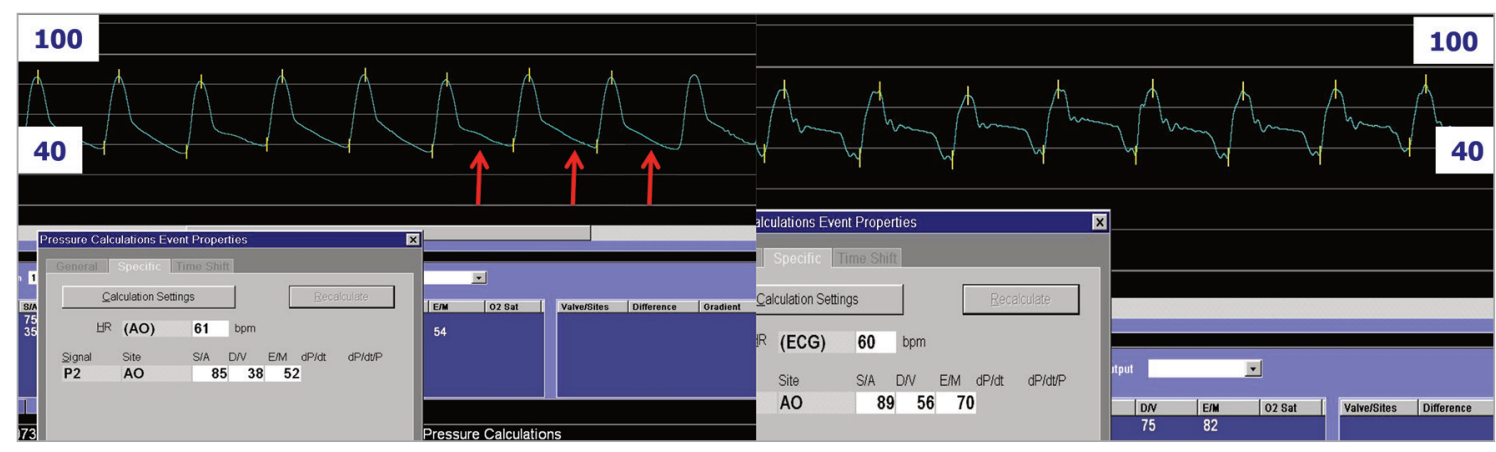

Figure 3. Pulsatile support with the PulseCath iVAC 2L. Left: pre support; right: with support. Note diastolic augmentation (red arrows) and resultant increase in mean arterial pressure. 


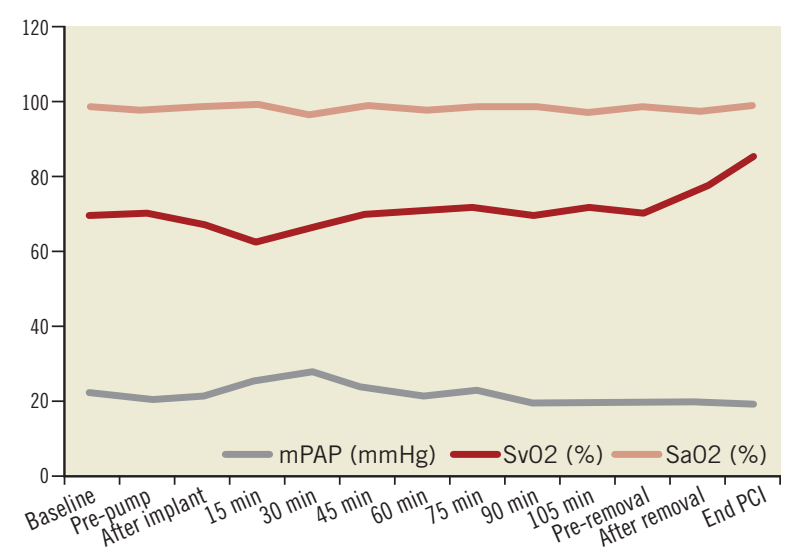

Figure 6. Change in mean pulmonary artery pressure (mPAP), arterial oxygen saturation ( $\mathrm{SaO2}$ ) and mixed venous oxygen saturation ( $\mathrm{SvO} 2)$.

Vascular Devices, Redwood City, CA, USA). A stiff 0.035" guidewire is inserted and an $18 \mathrm{Fr}$ arterial sheath, typically the SoloPath ${ }^{\mathrm{TM}}$ Balloon Expandable TransFemoral Access System (Terumo Corp., Tokyo, Japan), is navigated into the iliofemoral arterial trajectory. A 6 Fr pigtail catheter is used to cross the aortic valve and a pre-shaped 0.035" stiff guidewire (e.g., Amplatz Super Stiff ${ }^{\mathrm{TM}}$ Guidewire; Boston Scientific, Marlborough, MA, USA) is positioned in the apex of the left ventricle (Moving image 1).

\section{INTRODUCING THE IVAC 2L CATHETER}

The bi-directional flow catheter is pre-loaded with a dedicated dilator and advanced over the 0.035 " stiff guidewire until the stainless steel inlet tip has entered the left ventricle. The stiff guidewire and dilator are carefully removed, leaving the tip of the catheter in the LV apex. As the dilator is removed from the catheter, blood bleeds back and de-airs the system. The catheter is then temporarily squeezed with a surgical clamp. The chamber of the membrane pump is filled with heparinised saline. With a $60 \mathrm{~mL}$ syringe a wet-to-wet connection is made between the filled pump and the catheter. After confirmation of an air-free connection, the clamp is relieved and the IABP console is activated. The chamber will fill with arterialised blood. The pulsatile membrane mechanics can be visually monitored and assessed for thrombus formation. The target activated clotting time is between 250 and 300 seconds.

\section{RETRIEVAL}

At the end of the procedure the IABP console is switched off and the arterial tubing is clamped. With gradual continuous force the catheter can be retrieved from the body. Finally the 18 Fr SoloPath sheath can be removed and the arteriotomy closed with the initially constructed pre-closure.

\section{COMPLICATIONS}

As mentioned above, the requirement of an $18 \mathrm{Fr}$ arterial sheath with the PulseCath iVAC 2L mandates proper pre-procedural planning with imaging and meticulous access-site preparation to avoid

major access-site complications. Adequate per-procedural anticoagulation is essential to avoid thromboembolic phenomena but should be balanced with the risk of excessive bleeding. As with all LV support systems haemolysis should be monitored. Results from ongoing clinical studies will shed light on the incidence of devicespecific procedural complications.

\section{Conclusion}

The PulseCath iVAC 2L is a next-generation LVAD that can be safely introduced in a completely percutaneous fashion. It offers a pulsatile alternative to the continuous flow LV support systems and may have some theoretical benefits. It obtained CE mark approval for left ventricular circulatory support up to 24 hours on February 21st 2014. Table 1 displays indications and contraindications. A genuine IABP console drives the device. The assembly can generate an additional output of $1.5 \mathrm{~L} / \mathrm{min}$ on top of the existing cardiac output and, as such, significantly outperforms the IABP. Clinical studies to assess safety and efficacy are ongoing.

\section{Table 1. Indications and contraindications for use of PulseCath}

iVAC 2 L.

Indications

- Left ventricular (LV) mechanical circulatory support up to 24 hours

- During high-risk PCl

- Cardiogenic shock

\section{Impact on daily practice}

Percutaneous left ventricular assist devices are used to perform high-risk percutaneous coronary interventions and as an adjunct in the treatment of cardiogenic shock. The PulseCath iVAC $2 \mathrm{~L}$ is a next-generation pulsatile LVAD that is driven by a genuine intra-aortic balloon pump console and generates an additional output of $1.5 \mathrm{~L} / \mathrm{min}$ on top of the existing cardiac output. As such it outperforms the standard IABP and may be a valuable addition to the overall treatment armamentarium in patients requiring temporary circulatory support.

\section{Conflict of interest statement}

The authors have no conflicts of interest to declare. 


\section{References}

1. Perera D, Stables R, Clayton T, De Silva K, Lumley M, Clack L, Thomas M, Redwood S; BCIS-1 Investigators. Long-term mortality data from the balloon pump-assisted coronary intervention study (BCIS-1): a randomized, controlled trial of elective balloon counterpulsation during high-risk percutaneous coronary intervention. Circulation. 2013;127:207-12.

2. Peura JL, Colvin-Adams M, Francis GS, Grady KL, Hoffman TM, Jessup M, John R, Kiernan MS, Mitchell JE, O’Connell JB, Pagani FD, Petty M, Ravichandran P, Rogers JG, Semigran MJ, Toole JM; American Heart Association Heart Failure and Transplantation Committee of the Council on Clinical Cardiology; Council on Cardiopulmonary, Critical Care, Perioperative and Resuscitation; Council on Cardiovascular Disease in the Young; Council on Cardiovascular Nursing; Council on Cardiovascular Radiology and Intervention, and Council on Cardiovascular Surgery and Anesthesia. Recommendations for the use of mechanical circulatory support: device strategies and patient selection: a scientific statement from the American Heart Association. Circulation. 2012;126:2648-67.

3. Kar B, Basra SS, Shah NR, Loyalka P. Percutaneous circulatory support in cardiogenic shock: interventional bridge to recovery. Circulation. 2012;125:1809-17.

4. The Task Force on Myocardial Revascularization of the European Society of Cardiology (ESC) and the European Association for Cardio-Thoracic Surgery (EACTS), Wijns W, Kolh P, Danchin N, Di Mario C, Falk V, Folliguet T, Garg S, Huber K, James S, Knuuti J, Lopez-Sendon J, Marco J, Menicanti L, Ostojic M, Piepoli MF, Pirlet C, Pomar JL, Reifart N, Ribichini FL, Schalij MJ, Sergeant P, Serruys PW, Silber S, Sousa Uva M,
Taggart D. Guidelines on myocardial revascularization. Eur Heart J. 2010;31:2501-55.

5. Levine GN, Bates ER, Blankenship JC, Bailey SR, Bittl JA, Cercek B, Chambers CE, Ellis SG, Guyton RA, Hollenberg SM, Khot UN, Lange RA, Mauri L, Mehran R, Moussa ID, Mukherjee D, Nallamothu BK, Ting HH. 2011 ACCF/AHA/SCAI Guideline for Percutaneous Coronary Intervention: a report of the American College of Cardiology Foundation/American Heart Association Task Force on Practice Guidelines and the Society for Cardiovascular Angiography and Interventions. Circulation. 2011;124:e574-651.

6. Atluri P, Acker MA. Pulsatile left ventricular assist devices: what is the role in the modern era? Semin Thorac Cardiovasc Surg. 2010;22:106-8.

7. Scheidt S, Wilner G, Mueller H, Summers D, Lesch $M$, Wolff G, Krakauer J, Rubenfire M, Fleming P, Noon G, Oldham N, Killip T, Kantrowitz A. Intra-aortic balloon counterpulsation in cardiogenic shock. Report of a co-operative clinical trial. $N$ Engl $J$ Med. 1973;288:979-84.

8. Amico A, Brigiani MS, Vallabini A, Ferrante B, Marzovillo A, Loizzi D, Carbone C. PulseCath, a new short-term ventricular assist device: our experience in off-pump coronary artery bypass graft surgery. J Cardiovasc Med (Hagerstown). 2008;9:423-6.

\section{Supplementary data}

Moving image 1. PulseCath principle concept.

The supplementary data are published online at:

http://www.pcronline.com/

eurointervention/90th issue/168 